porous silicon for applications in the field of biochemistry detection. Porous silicon is ideal for the manufacture of transducers due to its spongelike morphology, characterized by a specific surface area of $500 \mathrm{~m} 2 \mathrm{~cm}-3$, which ensures an effective interaction with gases and liquids. Moreover, this porous material is inexpensive and fully compatible with standard microelectronic processes.

For this work, different structures of porous silicon were prepared from simple layers to multilayer (Bragg mirrors and optical microcavities), which have been characterized for use as transducers for optical detection of chemical substances and biological interactions.
An innovative optical system based on the measurement of reflectance in the visible range using self-sustaining porous silicon microcavities, for real time and labelfree studies of protein adsorption was developed. This study was complemented by the development of a theoretical model to study the kinetics of the diffusion and adsorption of proteins on porous silicon.

Moreover, macroporous silicon films sensitized with two proteins of interest for the detection of antibodies to T. cruzi (called CP1 and FRA) were prepared. The ultimate objective is to use these devices as platforms for detection of such antibodies present in hyperimmune sera obtained in rabbit.

\title{
Diversidad morfo-genética de Cercospora en soja. Detección precoz de la infección por C. kikuchii
}

\author{
Ma. Gabriela de los Milagros Latorre Rapela \\ latorrerapela@gmail.com \\ Dra. María Cristina Lurá \\ Dr. Iván S. Marcipar \\ Cátedra de Microbiología General \\ Facultad de Bioquímica y Ciencias Biológicas \\ Universidad Nacional del Litoral \\ Fecha de la defensa: 19/04/2013
}

\section{Resumen}

En la provincia de Santa Fe, el cultivo de la soja (Glycine max L. Merr.) representa una de las principales fuentes de ingreso. Entre los agentes causales de enfermedades infecciosas, las especies del género Cercospora constituyen un problema importante. El tizón de la hoja y la mancha púrpura de la semilla cuyo agente causal es $C$. kikuchii y la mancha ojo de rana (MOR) pro- ducida por C. sojina, reducen el valor nutricional de la planta y provocan severo deterioro de las cosechas.

Las especies de Cercospora producen una toxina de color rojo denominada cercosporina, que es la responsable de la patogenicidad y provoca la enfermedad. La producción de esta toxina es regulada por la proteína CFP ("Cercosporin Facilitator Protein") que, a su vez, es codificada por el gen cfp (cercosporin facilitator protein). La CFP, además, exporta la cercosporina hacia el exterior del hongo.

El propósito de este trabajo de tesis fue contribuir al conocimiento de las especies de Cercospora que ocasionaron patología en las plantas de soja, cultivadas en diferentes regiones de la provincia de Santa Fe durante las campañas 2005/2006, 
2006/2007, 2007/2008 y 2008/2009.

Para ello se plantearon como objetivos aislarlas y caracterizarlas fenotípica y molecularmente. La caracterización molecular se llevó a cabo mediante la amplificación de ISSR ("Inter Simple Sequence Repeats"), RAPD-PCR ("Random Amplified Polymorphic DNA") y la amplificación, por PCR, de regiones intergénicas (ITS) del ADNr ("Internally Transcribed Spacers"), las que se analizaron mediante RFLP-PCR ("Restriction Fragment-Length Polymorphisms") y se secuenciaron.

Se procesaron los pecíolos y folíolos de plantas con signos y síntomas visibles del tizón de la hoja y MOR. Se obtuvieron 52 aislamientos de los cuales, 42 provinieron de soja con tizón de la hoja y el resto de plantas enfermas con MOR. Se trabajó, además con las cepas de referencia Cercospora kikuchii NBRC 6711 y Cercospora sojina NBRC 6715.

Para la caracterización fenotípica se utilizaron diferentes medios de cultivo y se efectuaron observaciones macro y microscópicas, no detectándose mayores diferencias entre ambas especies. Al efectuar el examen directo al microscopio las estructuras de fructificación observadas fueron compatibles con C. kikuchii y C. sojina según correspondiera.

La amplificación de ISSR del genoma de cada aislamiento se llevó a cabo con los oligonucleótidos: $(\mathrm{CAC})_{5},(\mathrm{GTG})_{5}$ y $(\mathrm{GACA})_{4}$. Los dendrogramas generados permitieron reunir a los aislamientos regionales y a las cepas de referencia en grupos poco relacionados.

Otro de los objetivos planteados fue confeccionar un "mapa" delimitando regiones según las diferentes especies aisladas o los clones de una misma especie para lo cual, los grupos formados en base al dendrograma obtenido con (GTG) ${ }_{5}$, se volcaron sobre un plano de la provincia de Santa $\mathrm{Fe}$, detectándose una escasa asociación entre el origen geográfico de los hongos y la diversidad genética.

Las reacciones de RAPD se llevaron a cabo utilizando veinte oligonucleótidos de la serie OPA. El dendrograma mostró grandes distancias genéticas entre los diferentes hongos, detectándose un mayor grado de diversidad entre los aislamientos, lo que permitió corroborar la variabilidad fenotípica observada en los medios de cultivo. Según los resultados obtenidos, surgió como conclusión que las poblaciones argentinas de C. kikuchii y C. sojina son fenotípica, genotípica y geográficamente variables, constituyendo un grupo muy heterogéneo.

Para la amplificación de la región ITS del ADNr se utilizaron los oligonucleótidos ITS 4 e ITS 5. Los productos de PCR se digirieron con endonucleasas de restricción y se derivaron para su secuenciación. El alto grado de similitud del fragmento ITS y de los patrones RFLP observados entre las especies C. kikuchii y C. sojina aisladas, indicaron una alta homología en la secuencia de la región ITS estudiada, permitiendo confirmar que los hongos analizados, pertenecían al género Cercospora, no encontrándose diferencias entre las dos especies en estudio.

El último de los objetivos propuestos fue desarrollar métodos de detección precoz de especies de Cercospora en plantas de soja. Para cumplimentarlo se pusieron a punto diferentes técnicas para la detección del hongo en la planta y también se desarrolló un modelo experimental de infección de plantas de soja, por Cercospora. La técnica de Dot Blot permitió detectar la CFP en 
las plantas y la amplificación por PCR de un fragmento del gen cfp, permitió detectar el hongo en etapas tempranas de la infección por Cercospora.

Se concluye que los resultados obtenidos constituyen un aporte importante al conocimiento y a la detección temprana de dos de los hongos fitopatógenos más frecuentes que afectan uno de los cultivos más importantes para Argentina y, en particular, para la provincia de Santa Fe.

\section{Phenotypic and genotypic variability in}

Cercospora kikuchii in soybeans culture. early detection of $\mathrm{C}$. kikuchii infection

\section{Summary}

In Santa Fe province, soybean represents one of the greatest sources of revenue. Among the infectious disease-causing agents, fungi of the genus Cercospora have become a significant problem.

This Thesis attempts to contribute to the knowledge of Cercospora species which produced pathology in soybean plants grown in different regions of Santa Fe province during 2005/2006, 2006/2007, 2007/2008 and 2008/2009 campaigns.

The objectives of this work were the isolation and phenotypical characte- rization of the species, followed by a molecular characterization by means of ISSR ("Inter Simple Sequence Repeats"), RAPD-PCR ("Random Amplified Polymorphic DNA"), and PCR amplification of intergenic regions (ITS -"Internally Transcribed Spacers") of DNAr, which were analyzed by RFLP-PCR ("Restriction Fragment-Length Polymorphisms") and finally sequenced.

Petioles and leaflets of plants with visible signs and symptoms of leaf blight and frogeye leaf spot (MOR) were processed.

The Argentinian populations of C. kikuchii and C. sojina are phenotypically, genotypically and geographically variable, thus constituting a very heterogeneous group.

The high degree of similarity between the corresponding ITS fragments and the RFLP patterns of C. kikuchii and C. sojina isolates indicate a high homology in the ITS sequence under study.

Sequentiation results, only allowed confirming that the analyzed fungi belonged to the genus Cercospora, no differences being found between the two species under study.

The last of the objectives proposed was developing early detection methods of Cercospora species in soybean plants.

The Dot Blot and PCR techniques allowed to detect the fungus at the early stage of Cercospora infection.

\section{Factores que modulan la acción neurotrófica de los estrógenos en el hipocampo del adulto in vivo}

\author{
Guillermo Samuel Moreno-Piovano \\ gmoreno@fbcb.unl.edu.ar \\ Jorge Guillermo Ramos / Jorgelina Varayoud \\ Laboratorio de Endocrinología y Tumores
}

Hormonodependientes (LETH)

Facultad de Bioquímica y Ciencias Biológicas

Universidad Nacional del Litoral

Fecha de la defensa: 24/07/2013 